\title{
DEGREE REDUCTION OF BÉZIER CURVES USING CONSTRAINED CHEBYSHEV POLYNOMIALS OF THE SECOND KIND
}

\author{
YOUNG JOON AHN ${ }^{1}$
}

(Received 23 August, 2001; revised 7 June, 2002)

\begin{abstract}
In this paper a constrained Chebyshev polynomial of the second kind with $C^{1}$-continuity is proposed as an error function for degree reduction of Bézier curves with a $C^{\prime}$-constraint at both endpoints. A sharp upper bound of the $L_{\infty}$ norm for a constrained Chebyshev polynomial of the second kind with $C^{1}$-continuity can be obtained explicitly along with its coefficients, while those of the constrained Chebyshev polynomial which provides the best $C^{1}$-constrained degree reduction are obtained numerically. The representations in closed form for the coefficients and the error bound are very useful to the users of Computer Graphics or CAD/CAM systems. Using the error bound in the closed form, a simple subdivision scheme for $C^{1}$-constrained degree reduction within a given tolerance is presented. As an illustration, our method is applied to $C^{1}$-constrained degree reduction of a plane Bezier curve, and the numerical result is compared visually to that of the best degree reduction method.
\end{abstract}

\section{Introduction}

Degree reduction of Bézier curves is one of the most important problems in CAGD (Computer Aided Geometric Design) or CAD/CAM. In general, degree reduction cannot be done exactly, leading to approximation problems. Much effort has been directed at dealing with these problems in the past twenty years. Most publications focus on particular aspects of the problems, such as best degree reduction $[7,13]$, $C^{k}$-constraints $[1,2,10,14]$, the $L_{p}$-norm $[3,8,11,16-18]$ and simple algorithms $[12$, 19,21-23].

It is well-known that the error function of the best degree reduction in the uniform $\left(L_{\infty}\right)$ norm is the Chebyshev polynomial up to the leading coefficient. But, in many

\footnotetext{
${ }^{1}$ Department of Mathematics Education, Chosun University, Gwangju 501-759, Korea; e-mail: ahn@chosun.ac.kr.
}

(C) Australian Mathematical Society 2003, Serial-fee code 1446-1811/03 
actual CAD/CAM systems, it is required [2] that the approximate curve is continuous to order $k \geq 0$ at each junction point of consecutive curve segments. In order to reduce the degree of Bézier curves with $C^{0}, C^{1}$ or $C^{2}$-constraints at both endpoints, constrained Chebyshev polynomials are necessary as the error functions, which are the best approximate monic polynomials to zero $[13,14]$ for each case. In the $C^{0}$ constraint case, constrained Chebyshev polynomials can be expressed in terms of classical Chebyshev polynomials, but the other cases can be obtained numerically using the (modified) Remes algorithm [4, 22]. Recently, Kim and Ahn [10] proposed a good $C^{\mathbf{l}}$-constrained degree reduction method using constrained Jacobi polynomials. The method gives the coefficients of the polynomials explicitly, and also presents the $L_{\infty}$ norm of the polynomials in closed form for even degree.

In this paper, we propose another method for degree reduction with $C^{1}$-constraints using properly modified Chebyshev polynomials of the second kind. It is not the best $C^{1}$-constrained degree reduction and its uniform error bound is also larger than the $L_{\infty}$ norm of the constrained Jacobi polynomial with $C^{1}$-continuity numerically [10]. But our method presents the explicit form of a sharp error bound of the $L_{\infty}$ norm for all degrees along with the coefficients. We also present a simple subdivision scheme using the uniform error bound of our method in closed form. We apply our method to the degree reduction of a plane Bézier curve and compare the numerical result to that of the best degree reduction method.

The outline of this paper is as follows. In Section 2, we introduce our method of $C^{l}$-constrained degree reduction using constrained Chebyshev polynomials of the second kind with $C^{\mathbf{l}}$-continuity. We also present explicitly their uniform error bound and the control points in Bézier form, which are useful in actual CAD/CAM systems. Using the uniform error bound we give the subdivision scheme for the $C^{1}$-constrained degree reduction within a specified tolerance. In Section 3, we give an example of the $C^{1}$-constrained degree reduction of a plane Bézier curve of degree seven using our method, and compare its result to that of the best degree reduction by plotting the graphs of the degree reduced Bézier curves. In Section 4, we summarise our work.

\section{Degree reduction with $C^{1}$-constraints}

In this section, we introduce our method for degree reduction of a Bézier curve with a $C^{1}$-constraint at both endpoints. It is well-known [14] that the best $C^{1}$ - constrained degree reduction has the constrained Chebyshev polynomial with $C^{1}$-continuity as an error function, but the polynomial can be obtained numerically using the Remes algorithm. A good $C^{1}$-constrained degree reduction using the constrained Jacobi polynomial with $C^{1}$-continuity [10] has the error bound explicitly for even degree. We use constrained Chebyshev polynomial of the second kind with $C^{1}$-continuity 
as an error function for $C^{1}$-constrained degree reduction, since the polynomial has explicit representation in terms of Bézier coefficients and uniform error bound for all degrees. The following are some well-known properties for Chebyshev polynomials of the second kind which we shall call on later in the paper.

PROPERTY 2.1 (Refer to [6, 20]). The Chebyshev polynomial of the second kind, $U_{n}(x)=\sin ((n+1) \theta) / \sin (\theta), \theta=\arccos x$, of degree $n$, has the following properties:

(a) $U_{n}(x)$ has leading coefficient $2^{n}$;

(b) The zeros of $U_{n}(x)$ are $x=\cos (k \pi /(n+1)), k=1, \ldots, n$, and the largest zero of $U_{n}(x)$ is $\cos (\pi /(n+1))$ which is denoted by $\mu_{n}$ in this paper;

(c) $2^{-n} U_{n}(x)$ has the smallest $L_{\infty}$ norm on $[-1,1]$ amongst all monic polynomials weighted by $\sqrt{1-x^{2}}$;

(d) $U_{n}(x) \leq 1 / \sqrt{1-x^{2}}$ for $x \in[-1,1]$.

(e) $U_{n}(x)=\sum_{k=0}^{n} \Gamma_{k}^{n}((x-1) / 2)^{k}$, where

$$
\Gamma_{k}^{n}=4^{n}\left(\begin{array}{c}
n+1 / 2 \\
n-k
\end{array}\right)\left(\begin{array}{c}
n+k+1 \\
k
\end{array}\right) /\left(\begin{array}{c}
2 n+1 \\
n
\end{array}\right) .
$$

DEFINITION 2.2. We define the constrained Chebyshev polynomial of the second kind with $C^{1}$-continuity for $n \geq 4$ by

$$
E_{n}(t)=\frac{t(t-1) U_{n-2}\left(2 \mu_{n-2} t-\mu_{n-2}\right)}{\left(4 \mu_{n-2}\right)^{n-2}}
$$

for $t \in[0,1]$, where $m u_{n-2}=\cos (\pi /(n-1))$ is the largest zero of $U_{n-2}(x)$.

THEOREM 2.3. The constrained Chebyshev polynomial of the second kind with $C^{1}$ continuity $E_{n}(t)$ is a monic polynomial of degree $n$, has double zeros at $t=0,1$, and its uniform norm is bounded by

$$
\left\|E_{n}(\cdot)\right\|_{L_{\infty}[0,1]} \leq \frac{1}{4^{n-1} \cos ^{n-2}(\pi /(n-1))} .
$$

In particular, equality holds for even $n$.

ProOF. By Property 2.1 (a), $U_{n-2}\left(2 \mu_{n-2} t-\mu_{n-2}\right)$ has leading coefficient $\left(4 \mu_{n-2}\right)^{n-2}$ so that $E_{n}(t)$ is a monic polynomial.

By Property $2.1(\mathrm{~b}), U_{n-2}(x)$ has a zero at $\pm \mu_{n-2}$, so that $E_{n}(t)$ has double zeros at both endpoints $t=0,1$. 


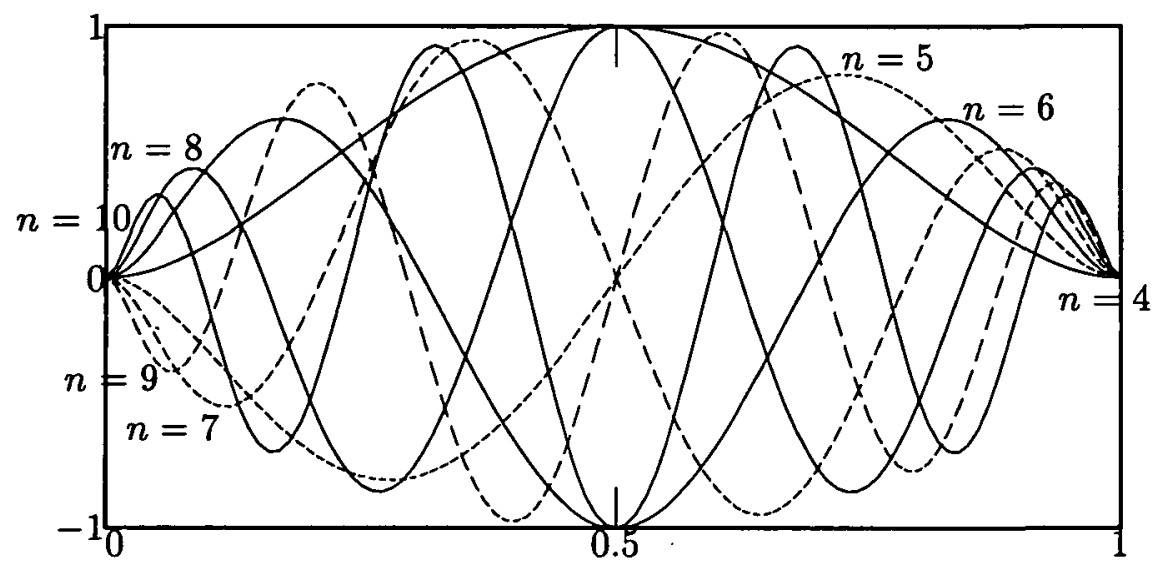

FIGURE 1. Constrained Chebyshev polynomials of the second kind with $C^{1}$-continuity, $4^{n-1} \cos ^{n-2}(\pi /(n-$ 1)) $E_{n}(t)$, uniformised by the error bound, for $4 \leq n \leq 10$, are plotted by dashed lines for odd degree $n$ and by solid lines for even $n$.

By Property 2.1 (d), we have

$$
\left|E_{n}(t)\right| \leq \frac{1}{\left(4 \mu_{n-2}\right)^{n-2}} \frac{t(1-t)}{\sqrt{1-\left(2 \mu_{n-2} t-\mu_{n-2}\right)^{2}}},
$$

for all $t \in[0,1]$. Since $0<\mu_{n-2}<1$, we get

$$
4 t(1-t)=\left(1-(2 t-1)^{2}\right) \leq\left(1-\mu_{n-2}^{2}(2 t-1)^{2}\right) \leq \sqrt{1-\mu_{n-2}^{2}(2 t-1)^{2}}
$$

for all $t \in[0,1]$. Thus

$$
\left|E_{n}(t)\right| \leq \frac{1}{\left(4 \mu_{n-2}\right)^{n-2}} \cdot \frac{1}{4}=\frac{1}{4^{n-1} \cos ^{n-2}(\pi /(n-1))}
$$

for all $t \in[0,1]$, and (2.1) is satisfied. Particularly, for even $n$,

$$
E_{n}(1 / 2)=\frac{1}{4} \frac{U_{n-2}(0)}{\left(4 \mu_{n-2}\right)^{n-2}}=\frac{1}{4^{n-1} \cos ^{n-2}(\pi /(n-1))}
$$

yields that the equality in (2.1) holds.

As an illustration, we plot the constrained Chebyshev polynomial of the second kind with $C^{1}$-continuity, $4^{n-1} \cos ^{n-2}(\pi /(n-1)) E_{n}(t)$, uniformised by the error bound in Theorem 2.3, for $4 \leq n \leq 10$, in Figure 1. As shown in Table 1, we compare the uniform error bound of $E_{n}(t)$ which is obtainable explicitly with the uniform 
TABLE 1. The uniform error norms of the best degree reduction with $C^{l}$-constraint $T_{n}^{(2)}(t)$ obtained by the Remes algorithm, and the uniform error bound of our method $E_{n}(t)$ obtained explicitly.

\begin{tabular}{|clc|}
\hline degree $n$ & $\left\|C_{n}^{2}(\cdot)\right\|_{L_{\infty}[0.11}$ & bound of $\left\|E_{n}(\cdot)\right\|_{\left.L_{\infty} \mid 0.1\right]}$ \\
\hline \hline 4 & $6.2500 \times 10^{-2}$ & $6.2500 \times 10^{-2}$ \\
\hline 5 & $8.9443 \times 10^{-3}$ & $1.1049 \times 10^{-2}$ \\
\hline 6 & $1.6546 \times 10^{-3}$ & $2.2797 \times 10^{-3}$ \\
\hline 7 & $3.4009 \times 10^{-4}$ & $5.0117 \times 10^{-4}$ \\
\hline 8 & $7.3959 \times 10^{-5}$ & $1.1411 \times 10^{-4}$ \\
\hline 9 & $1.6648 \times 10^{-5}$ & $2.6559 \times 10^{-5}$ \\
\hline 10 & $3.8340 \times 10^{-6}$ & $6.2744 \times 10^{-6}$ \\
\hline 11 & $8.9712 \times 10^{-7}$ & $1.4981 \times 10^{-6}$ \\
\hline 12 & $2.1238 \times 10^{-7}$ & $3.6051 \times 10^{-7}$ \\
\hline 13 & $5.0724 \times 10^{-8}$ & $8.7277 \times 10^{-8}$ \\
\hline 14 & $1.2196 \times 10^{-8}$ & $2.1228 \times 10^{-8}$ \\
\hline 15 & $2.9482 \times 10^{-9}$ & $5.1822 \times 10^{-9}$ \\
\hline 16 & $7.1577 \times 10^{-10}$ & $1.2689 \times 10^{-9}$ \\
\hline 17 & $1.7440 \times 10^{-10}$ & $3.1148 \times 10^{-10}$ \\
\hline 18 & $4.2614 \times 10^{-11}$ & $7.662 \times 10^{-11}$ \\
\hline 19 & $1.0440 \times 10^{-11}$ & $1.8878 \times 10^{-11}$ \\
\hline 20 & $2.5628 \times 10^{-12}$ & $4.6581 \times 10^{-12}$ \\
\hline
\end{tabular}

norm of the constrained Chebyshev polynomials with $C^{\prime}$-continuity $C_{n}^{2}(t)$ of leading coefficient 1 which are obtained numerically by the Remes algorithm [14]. (The superscript 2 on $C_{n}^{2}(t)$ means double zeros at both endpoints $[10,14]$.)

Now, using the constrained Chebyshev polynomial of the second kind with $C^{\mathbf{l}}$ continuity $E_{n}(t)$, we describe our method of $C^{1}$-constrained degree reduction of a Bézier curve $f(t)=\sum_{i=0}^{n} b_{i} B_{i}^{n}(t)$ of degree $n$, where $B_{i}^{n}(t)=\left(\begin{array}{l}n \\ i\end{array}\right) t^{i}(1-t)^{n-i}$ and $b_{i}$, for $i=0, \ldots, n$, are Bernstein polynomials and Bézier coefficients (or control points), respectively.

PROPOSITION 2.4. Let $f(t)=\sum_{i=0}^{n} b_{i} B_{i}^{n}(t)$ be the given Bézier curve of degree $n$ having control points $b_{i}$. Then the Bézier curve $\vec{f}(t)$ of degree (less than or equal to) $n-1$ given by $\bar{f}(t):=f(t)-\Delta^{n} b_{0} E_{n}(t)$ is a degree reduction of $f(t)$ with $C^{\prime}$-constraint at both endpoints, and its error norm is bounded by

$$
\|f(\cdot)-\bar{f}(\cdot)\|_{L_{\infty}[0,1]}=\left|\Delta^{n} b_{0}\right|\left\|E_{n}(\cdot)\right\|_{L_{\infty}[0,1]} \leq \frac{\left|\Delta^{n} b_{0}\right|}{4^{n-1} \cos ^{n-2}(\pi /(n-1))},
$$

where the nth forward difference $\Delta^{n} b_{0}=\sum_{i=0}^{n}(-1)^{i}\left(\begin{array}{c}n \\ i\end{array}\right) b_{n-i}$ is equal to the leading coefficient of the polynomial $f(t)$ of degree $n$.

PROOF. Since the $n$-th degree polynomials $f(t)$ and $\Delta^{n} b_{0} E_{n}(t)$ have the same 
leading coefficient, $\bar{f}(t)$ is a polynomial of degree less than or equal to $n-1$. The $k$-th order derivative $E_{n}^{(k)}(t)=0$ for $k=0,1$, at $t=0,1$, yields that $\bar{f}^{(k)}(t)=f^{(k)}(t)$ and $\bar{f}(t)$ is a degree reduction of $f(t)$ with a $C^{1}$-constraint at both endpoints. The error bound is easily obtained from Theorem 2.3 .

The following proposition gives the closed form of the control points of the constrained Chebyshev polynomials of the second kind with $C^{1}$-continuity in Bézier form, which is needed to calculate $\bar{f}(t)$ as a Bézier curve or a segment of spline in CAD/CAM systems.

PROPOSITION 2.5. The constrained Chebyshev polynomial of the second kind with $C^{1}$-continuity of degree $n$ in Bézier form is given by

$$
E_{n}(t)=\sum_{i=0}^{n} c_{i} B_{i}^{n}(t)
$$

where $c_{0}=c_{1}=c_{n-1}=c_{n}=0$ and

$$
c_{i}=\sum_{j=0}^{i-1} \sum_{k=j}^{n-2}-\frac{\left(\begin{array}{c}
n-3 / 2 \\
n-2-k
\end{array}\right)\left(\begin{array}{c}
n+k-1 \\
k
\end{array}\right)\left(\begin{array}{c}
n-2-j \\
i-1-j
\end{array}\right)\left(\begin{array}{l}
k \\
j
\end{array}\right)}{\left(\begin{array}{c}
2 n-3 \\
n-2
\end{array}\right)\left(\begin{array}{l}
n \\
i
\end{array}\right)}\left(\frac{-\mu_{n-2}-1}{2}\right)^{k-j} \mu_{n-2}^{j+2-n}
$$

$i=2, \ldots, n-2$.

Proof. By Definition 2.2 and Property $2.1(\mathrm{e})$, we have $E_{n}(t)$ in a power basis

$$
\begin{aligned}
E_{n}(t) & =\frac{t(t-1)}{\left(4 \mu_{n-2}\right)^{n-2}} \sum_{k=0}^{n-2} \Gamma_{k}^{n-2}\left(\frac{2 \mu_{n-2} t-\mu_{n-1}-1}{2}\right)^{k} \\
& =\frac{t(t-1)}{\left(4 \mu_{n-2}\right)^{n-2}} \sum_{k=0}^{n-2} \sum_{j=0}^{k} \Gamma_{k}^{n-2}\left(\begin{array}{l}
k \\
j
\end{array}\right)\left(\frac{-\mu_{n-2}-1}{2}\right)^{k-j} \mu_{n-2}^{j} t^{j} \\
& =\frac{t(t-1)}{4^{n-2}} \sum_{j=0}^{n-2} \sum_{k=j}^{n-2} \Gamma_{k}^{n-2}\left(\begin{array}{l}
k \\
j
\end{array}\right)\left(\frac{-\mu_{n-2}-1}{2}\right)^{k-j} \mu_{n-2}^{j+2-n} t^{j}
\end{aligned}
$$

for $n \geq 2$. Using the transformation from a power basis of degree $m$ into a Bernstein basis

$$
\sum_{j=0}^{m} v_{j} t^{j}=\sum_{i=0}^{m} \sum_{j=0}^{i} \frac{\left(\begin{array}{c}
m-j \\
i-j
\end{array}\right)}{\left(\begin{array}{c}
m \\
i
\end{array}\right)} v_{j} B_{i}^{m}(t)
$$

we obtain

$$
E_{n}(t)=\frac{t(t-1)}{4^{n-2}} \sum_{i=0}^{n-2} \sum_{j=0}^{i} \sum_{k=j}^{n-2} \frac{\left(\begin{array}{c}
n-2-j \\
i-j
\end{array}\right)}{\left(\begin{array}{c}
n-2 \\
i
\end{array}\right)} \Gamma_{k}^{n-2}\left(\begin{array}{l}
k \\
j
\end{array}\right)\left(\frac{-\mu_{n-2}-1}{2}\right)^{k-j} \mu_{n-2}^{j+2-n} B_{i}^{n-2}(t)
$$


By the equation

$$
t(t-1) \sum_{i=0}^{n-2} u_{i} B_{i}^{n-2}(t)=-\sum_{i=1}^{n-1} \frac{\left(\begin{array}{c}
n-2 \\
i-1
\end{array}\right)}{\left(\begin{array}{l}
n \\
i
\end{array}\right)} u_{i-1} B_{i}^{n}(t)
$$

we have the Bézier form of the constrained Chebyshev polynomial of the second kind with $C^{1}$-continuity

$$
E_{n}(t)=\frac{-1}{4^{n-2}} \sum_{i=1}^{n-1} \sum_{j=0}^{i-1} \sum_{k=j}^{n-2} \frac{\left(\begin{array}{c}
n-2-j \\
i-1-j
\end{array}\right)}{\left(\begin{array}{l}
n \\
i
\end{array}\right)} \Gamma_{k}^{n-2}\left(\begin{array}{l}
k \\
j
\end{array}\right)\left(\frac{-\mu_{n-2}-1}{2}\right)^{k-j} \mu_{n-2}^{j+2-n} B_{i}^{n}(t)
$$

Thus (2.2), for $i=2, \ldots, n-2$, follows from (2.3) and Property 2.1 (e), and $c_{0}=c_{1}=c_{n-1}=c_{n}=0$ follows from the fact that $E_{n}(t)$ has double zeros at $t=0,1$.

The fact that $c_{1}=c_{n-1}=0$ can be also proved by calculating (2.3) as follows:

$$
\begin{aligned}
c_{1} & =\frac{-1}{n 4^{n-2}} \sum_{k=0}^{n-2} \Gamma_{k}^{n-2}\left(\frac{-\mu_{n-2}-1}{2}\right)^{k} \mu_{n-2}^{2-n}=\frac{-1}{n\left(4 \mu_{n-2}\right)^{n-2}} U_{n-2}\left(-\mu_{n-2}\right)=0, \\
c_{n-1} & =\frac{-1}{n 4^{n-2}} \sum_{j=0}^{n-2} \sum_{k=j}^{n-2} \Gamma_{k}^{n-2}\left(\begin{array}{l}
k \\
j
\end{array}\right)\left(\frac{-\mu_{n-2}-1}{2}\right)^{k-j} \mu_{n-2}^{j+2-n} \\
& =\frac{-1}{n\left(4 \mu_{n-2}\right)^{n-2}} \sum_{k=0}^{n-2} U_{n-2}\left(\mu_{n-2}\right)=0 .
\end{aligned}
$$

Using the proposition above we represent the degree reduction $\tilde{f}(t)$ of $f(t)$ in Bézier form:

$$
\bar{f}(t)=\sum_{i=0}^{n-1} \bar{b}_{i} B_{i}^{n-1}(t)
$$

where $\bar{b}_{i}, i=0, \ldots, n-1$, are the Bézier coefficients of $\bar{f}(t)$ of degree $n-1$.

PROPOSITION 2.6. The Bézier coefficients $\bar{b}_{i}$ of $\bar{f}(t)$ are given by

$$
\bar{b}_{i}= \begin{cases}b_{0}-\Delta^{n} b_{0} c_{0} & \text { if } i=0 \\ \frac{n}{n-i}\left(b_{i}-\Delta^{n} b_{0} c_{i}-\frac{i}{n} \bar{b}_{i-1}\right) & \text { if } i=1, \ldots, n-1,\end{cases}
$$

recursively.

PROOF. See Kim and Ahn [10]. 
In the practical application, for a given tolerance it is necessary to subdivide the Bézier curve $f(t)$ of degree $n$ into $k$ pieces in order to approximate each piece by the lower degree Bézier curve within the tolerance. In the following theorem, we show how many subdivisions are required so that each piecewise degree reduction using our method has error less than the given tolerance.

THEOREM 2.7. For a given tolerance $\varepsilon$, the Bézier curve $f(t)=\sum_{i=0}^{n} b_{i} B_{i}^{n}(t)$ must be subdivided into $k$ segments so that the degree reduction for each segment has uniform error less than $\varepsilon$, where $k$ is given by

$$
k=\left\lceil\left(\frac{\left|\Delta^{n} b_{0}\right|}{\varepsilon 4^{n-1} \cos ^{n-2}(\pi /(n-1))}\right)^{1 / n}\right\rceil
$$

and $\lceil x\rceil$ denotes the smallest integer larger than $x$.

Proof. Since each subdivided segment $f^{j}(t)=f(t / k+j / k), j=0, \ldots, k-1$, has leading coefficient $(1 / k)^{n} \Delta^{n} b_{0}$, the uniform error of the degree reduction for each segment is equal to $(1 / k)^{n}\left|\Delta^{n} b_{0}\right||| E_{n}(\cdot) \|_{L_{\infty}[0,1]}$. In order to satisfy that the uniform error bound be less than $\varepsilon,(2.4)$ holds by Theorem 2.3 .

\section{Example}

In this section, we apply our method to reduce the degree of a plane Bézier curve of degree seven. Let the Bézier curve be given by [7]

$$
f(t)=\sum_{i=0}^{7} \mathbf{b}_{i} B_{i}^{7}(t)
$$

where the $\mathbf{b}_{i}$ 's are $(0,0),(.5,0),(.3,-1),(1, .25),(1,-.75),(1.7, .25),(1.5,-.5)$, $(2,-.5)$, in order, as shown in Figure 2 . The degree reduction method using a constrained Chebyshev polynomial of the second kind with $C^{1}$-continuity yields the approximate Bézier curve $\bar{f}(t)$ of degree six,

$$
\bar{f}(t)=f(t)-\Delta^{7} \mathbf{b}_{0} E_{7}(t)=\sum_{i=0}^{6} \bar{b}_{i} B_{i}^{6}(t),
$$

where $\Delta^{7} \mathbf{b}_{0}=(24.4,64.25)$ and the $\bar{b}_{i}$ 's are $(0,0),(0.583,0),(0.322,-1.043)$, $(1,-0.119),(1.678,0.193),(1.4 .17,-0.5),(2 .,-0.5)$, in order. The uniform error bound for the degree reduction is given by

$$
\|f(\cdot)-\bar{f}(\cdot)\|_{L_{\infty}[0,1]} \leq|(24.4,64.25)|\left\|E_{7}(\cdot)\right\|_{L_{\infty}[0,1]} \approx 0.0336 .
$$




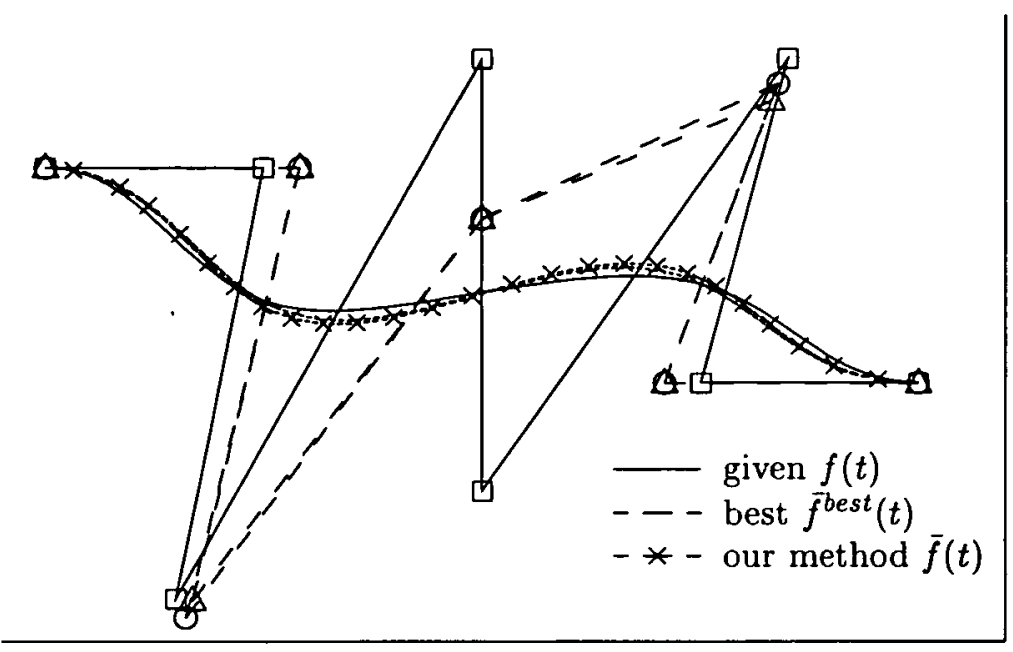

FIGURE 2. The best degree reduction and our method: the given Bézier curve $f(t)$, the best degree reduction $\bar{f}^{\text {best }}(t)$ and our method $\bar{f}(t)$ are plotted by solid lines, dashed lines, and dashed lines with crosses, respectively. The boxes, triangles and circles are the control points of each Bézier curve, in order.

On the other hand, the best degree reduction by the constrained Chebyshev polynomial yields $f^{\text {best }}(t)=f(t)-(24.4,64.25) C_{7}^{2}(t)=\sum_{i=0}^{6} v_{i} B_{i}^{6}(t)$, where the $v_{i}$ 's are $(0,0),(0.583333,0),(0.337096,-1.00389),(1,-0.11875),(1.6629,0.153889)$, $(1.41667,-0.5),(2 .,-0.5)$, in order. We compare the graph of our degree reduction $\bar{f}(t)$ to that of $\bar{f}^{\text {best }}(t)$ by plotting the Bézier curves and their control points in Figure 2.

Let the error tolerance $\varepsilon$ be given by 0.001 . By Theorem 2.7, the curve $f(t)$ must be subdivided into two pieces because

$$
k=\left\lceil\left(\frac{|(24.4,62.5)|}{4^{6}(\cos (\pi / 6))^{5} \times 0.001}\right)^{1 / 7}\right\rceil \approx\lceil 1.65\rceil=2 .
$$

By subdividing $f(t)$ at $t=1 / 2$ into two Bézier segments as shown in Figure 3, the uniform error bound is given by

$$
\frac{1}{2^{7}} \times \frac{|(24.4,64.25)|}{4^{6} \cos (\pi / 6)^{5}} \approx 0.000263<0.001
$$

and the degree reduction with $C^{1}$-constraint for each Bézier segment is achieved within the specified tolerance. 


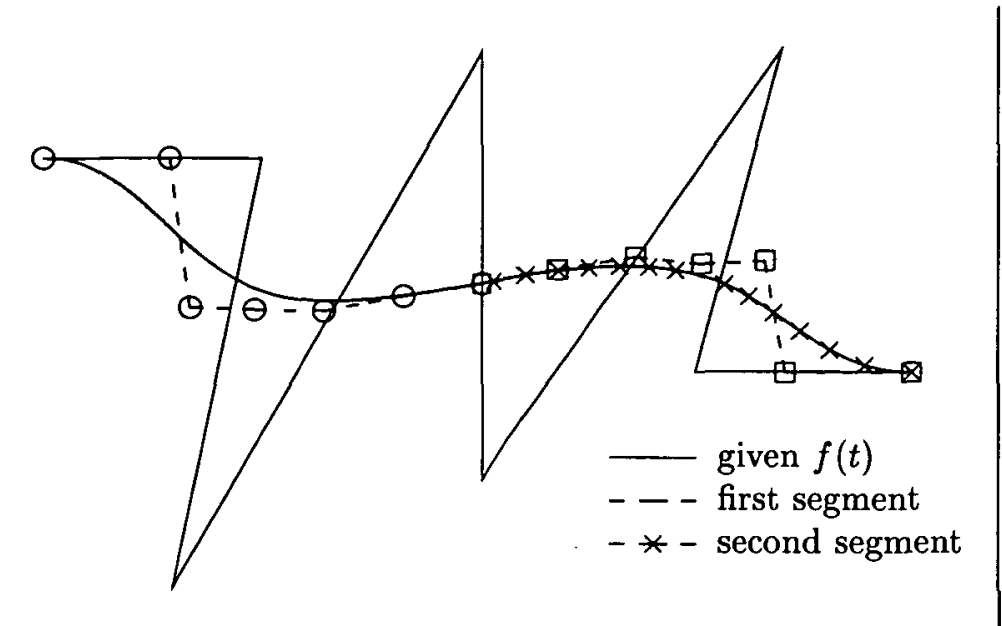

FIGURE 3. Degree reduction using the subdivision scheme: the degree reductions using our method $E_{n}(t)$ for the first and second segments are plotted by dashed lines and dashed lines with crosses, respectively. The circles and boxes are the control points of the degree reduction for each subdivision Bézier segment, in order.

\section{Comments}

In this paper we presented a method for degree reduction of Bézier curves with the $C^{1}$-constraint at both endpoints having the explicit form of the uniform error bound. The constrained Chebyshev polynomial of the second kind with $C^{1}$-continuity $E_{n}(t)$ proposed in this paper has the minimum $L_{\infty}$ error bound among the monic polynomials which have zeros of multiplicity two at both endpoints $t=0,1$, and for which the uniform error bounds are known explicitly. Even if $E_{n}(t)$ is not the best degree reduction with a $C^{1}$-constraint and its error bound is larger than that of the constrained Jacobi polynomial with $C^{1}$-continuity proposed by Kim and Ahn [10], it is more useful than those polynomials since no numerical calculations are needed. That is to say, the Bézier coefficients and the uniform error bound of $E_{n}(t)$ in explicit form can be obtained for any degree. We also gave a simple subdivision scheme and the numerical results for an example using our degree reduction method.

\section{Acknowledgements}

The author is very grateful to the Editor and anonymous referees for their valuable comments. This study was supported by research funds from Chosun University, 2003. 


\section{References}

[1] Y. J. Ahn, "Geometric conic spline approximation in CAGD", Commun. Korean Math. Soc. 17 (2002) 331-347.

[2] P. Bogaki, S. E. Weinstein and Y. Xu, "Degree reduction of Bézier curves by uniform approximation with endpoint interpolation", Comput. Aided Design 27 (1995) 651-662.

[3] G. Brunnett, T. Schreiber and J. Braun, "The geometry of optimal degree reduction of Bézier curves", Comput. Aided Geom. Design 13 (1996) 773-788.

[4] E. W. Cheney, Introduction to approximation theory (Chelsea, New York, 1982).

[5] T. S. Chihara, An introduction to orthogonal polynomials (Gordon and Breach, New York, 1978).

[6] P. J. Davis, Interpolation and approximation (Dover, New York, 1975).

[7] M. Eck, "Degree reduction of Bézier curves", Comput. Aided Geom. Design 10 (1993) 237-251.

[8] M. Eck, "Least squares degree reduction of Bézier curves", Comput. Aided Design 27 (1995) 845-852.

[9] G. Farin, Curves and surfaces for computer aided geometric design (Academic Press, Boston, MA, 1993).

[10] H. J. Kim and Y. J. Ahn, "Good degree reduction of Bézier curves using constrained Jacobi polynomials", Comput. Math. Appl. 40 (2000) 1205-1215.

[11] H. O. Kim, J. H. Kim and S. Y. Moon, "Degree reduction of Bézier curves and filter banks", Comput. Math. Appl. 31 (1996) 23-30.

[12] H. O. Kim and S. Y. Moon, "Degree reduction of Bézier curves by $L^{1}$-approximation with endpoint interpolation", Comput. Math. Appl. 33 (1997) 67-77.

[13] M. A. Lachance, "Chebyshev economization for parametric surfaces", Comput. Aided Geom. Design 5 (1988) 195-208.

[14] M. A. Lachance, "Approximation by constrained parametric polynomials", Rocky Mountain J. Math. 21 (1991) 473-488.

[15] G. G. Lorentz, Approximation functions (Chelsea, New York, 1986).

[16] D. Lutterkort, J. Peters and U. Reif, "Polynomial degree reduction in the $L_{2}$-norm equals best Euclidean approximation of Bézier coefficients", Comput. Aided Geom. Design 16 (1999) 607612.

[17] Y. Park and B. G. Lee, "Distance for Bézier curves and degree reduction", Bull. Austral. Math. Soc. 56 (1997) 507-515.

[18] J. Peters and U. Reif, "Least squares approximation of Bézier coefficients provides best degree reduction in the $L_{2}$-norm", J. Approx. Theory 104 (2000) 90-97.

[19] L. Piegl and W. Tiller, "Algorithm for degree reduction of B-spline curves", Comput. Aided Design 27 (1995) 101-110.

[20] G. Szegö, Orthogonal polynomials, AMS Coll. Publ. 23 (Amer. Math. Soc., Providence, RI, 1975).

[21] M. A. Watkins and A. J. Worsey, "Degree reduction of Bézier curves", Comput. Aided Design 20 (1988) 398-405.

[22] G. A. Watson, Approximation theory and numerical methods (Wiley, New York, 1980).

[23] J. H. Yong, S. M. Hu, J. G. Sun and X. Y. Tan, "Degree reduction of B-spline curves", Comput. Aided Geom. Design 18 (2001) 117-127. 